\title{
A Case of Amniotic Fluid Embolism Postdelivery: Catastrophe averted Successfully
}

\author{
${ }^{1}$ Priyanka Mehta, ${ }^{2}$ Lalitha Devadason, ${ }^{3}$ Jaya Vijayaraghavan
}

\begin{abstract}
Amniotic fluid embolism (AFE) is a rare and often fatal obstetric condition, characterized by sudden cardiovascular collapse, altered mental status, and disseminated intravascular coagulation (DIC). The disease is rare, with an incidence ranging from one in 600 to one in 80,000 , perhaps because there is no established laboratory marker diagnostic suitable for both survivors and fatalities alike. We present a case of AFE in a low-risk primigravida which was successfully managed by a multidisciplinary team.
\end{abstract}

Keywords: Amniotic fluid embolism, Coagulopathy, Hypoxia, Cardiovascular collapse, Maternal death.

How to cite this article: Mehta P, Devadason L, Vijayaraghavan J. A Case of Amniotic Fluid Embolism Postdelivery: Catastrophe averted Successfully. J South Asian Feder Obst Gynae 2014; 6(3):199-201.

\section{Source of support: Nil}

Conflict of interest: None

\section{INTRODUCTION}

A 28-year-old primigravida had an uneventful normal delivery after prostaglandin induction of labor for prelabor rupture of membranes (PROM). Labor was uneventful apart from meconium in the hindwaters. In the 10 minutes following delivery, she had sudden unexplained air hunger and breathlessness. She had sudden cardiovascular collapse, pulse was feeble 130 per minute, blood pressure $80 / 40 \mathrm{~mm} \mathrm{Hg}$. Amniotic fluid embolism was suspected, she was resuscitated with crystalloids, ventilatory support given. Coagulation profile was deranged, INR 2 following which she had postpartum hemorrhage. In spite of repeated doses of oxytocin, methergine, prostaglandin F2a, intrauterine bakri balloon tamponade, she continued to bleed.

Arterial blood gases showed hypoxia and hypoxemia. Surgical management initiated in view of uncontrolled

\footnotetext{
${ }^{1}$ Assistant Professor, ${ }^{2}$ Professor, ${ }^{3}$ Professor and Head

${ }^{1-3}$ Department of Obstetrics and Gynecology, Sri Ramachandra Medical College, Chennai, Tamil Nadu, India
}

Corresponding Author: Priyanka Mehta, A-1, 33/12, Cee Dee Yes Apartments, VOC Main Road, Kodambakkam Chennai-600024, Tamil Nadu, India, Phone: 09894425623 e-mail: priyankavimal6@gmail.com hemorrhage and shock. Multidisciplinary team including anesthetist, hematologist and senior obstetrician was involved. Patient was on ventilatory support.

Subtotal hysterectomy (Fig. 1) and uterine artery embolization was done simultaneously in the cath lab with help of the team of intervention radiologists. Patient was weaned from ventilator 24 hours later. A total of 14 packed cells, 19 fresh frozen plasma and 14 platelets were transfused during the process of resuscitation. Acute kidney injury developed in postoperative period which resolved with no sequelae. She was discharged on 10th postoperative day. On review 1 month later, she had weakness of the lower limbs for which supportive treatment was given.

\section{DISCUSSION}

Amniotic fluid embolism (AFE) is a rare and often fatal obstetric condition, characterized by sudden cardiovascular collapse, altered mental status, and disseminated intravascular coagulation (DIC). The presence of fetal debris in the pulmonary blood vessels of a mother who had died suddenly in labor was first described by Meyer ${ }^{1}$ in 1926, but it was not until 1941 that death following sudden collapse during labor or in the immediate postpartum period was attributed to AFE. ${ }^{2}$

With the mechanism remaining to be elucidated, AFE remains a clinical diagnosis. It is diagnosed based on one or more of four key signs/symptoms: cardiovascular collapse, respiratory distress, coagulopathy, and/or coma/seizures. ${ }^{3}$ The only laboratory test that reliably supports the diagnosis is the finding of fetal material in the maternal pulmonary circulation at autopsy.

Several factors have been associated with amniotic fluid embolism syndrome. ${ }^{4}$ They include precipitous or tumultuous labor, advanced maternal age, cesarean and instrumental delivery, placenta previa and abruption, grand multiparity ( $\geq 5$ live births or stillbirths), cervical lacerations, fetal distress, eclampsia, and medical induction of labor. ${ }^{5}$ Our understanding of the pathogenesis suggests that these factors probably are associated with amniotic fluid embolism syndrome, but not the direct cause. The syndrome is best considered unpredictable and unpreventable. 


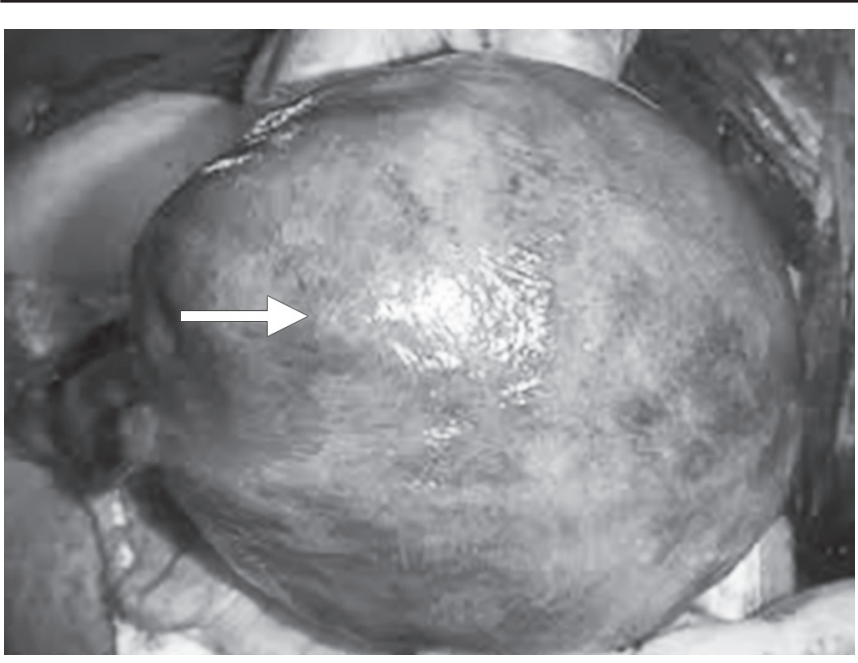

Fig. 1: Atonic uterus needing hysterectomy

Amniotic fluid probably enters the maternal circulation through the endocervical veins, the placental insertion site, or a site of uterine trauma. Once it reaches the maternal circulation, it can precipitate cardiogenic shock, respiratory failure, and, most likely, an inflammatory and anaphylactoid response.

The major clinical findings are the abrupt and fulminant onset of hypotension due to cardiogenic shock, hypoxemia and respiratory failure, disseminated intravascular coagulation, coma or seizures. ${ }^{12}$

Pulmonary hypertension and right-heart strain/ failure may be the result of physical amniotic fluid debris in the pulmonary vasculature. Rapid decline in pulse oximetry values or sudden absence or decrease in endtidal carbon dioxide. Ventilation-perfusion mismatching due to pulmonary vascular constriction at the onset of AFE explains the sudden hypoxia and respiratory arrest.

Myocardial failure is in response to sudden pulmonary hypertension. Direct myocardial depressant effect of humoral mediators in amniotic fluid and hypoxemia cause myocardial dysfunction. The intravascular entry of procoagulant and anticoagulant factors of amniotic fluid may disrupt the coagulation balance of pregnancy. Encephalopathy is thought to be secondary to hypoxia. As many as $85 \%$ of AFE survivors have been reported to have residual neurologic deficits.

The first theory is that a tumultuous labor, abnormal placentation, surgical trauma, or any other breach of the barrier between maternal blood and amniotic fluid allows the forced entry of amniotic fluid into the systemic circulation and results in a physical obstruction of the pulmonary circulation. The second hypothesis suggests that entry of amniotic fluid into the maternal circulation activates inflammatory mediators, causing a immunologic response. The direct procoagulant property of amniotic fluid may explain the prevalence of DIC in
AFE. An alternative explanation for DIC is an immunemediated response producing complement activation. ${ }^{7}$

The hemodynamic response in AFE is biphasic, with initial pulmonary hypertension and right ventricular failure, followed by left ventricular failure.

Understanding its etiology and pathophysiology is incomplete, and the criteria used to make its diagnosis are controversial. ${ }^{8}$

In the absence of other medical explanations, the peripartum patient who has any combination of acute hemodynamic collapse, respiratory distress/hypoxia, DIC, and/or mental status changes should be considered to possibly have AFE.

Survival after AFE has improved significantly with early recognition of this syndrome and prompt and early resuscitative measures. The decrease in the mortality rate results solely from early diagnosis and prompt treatment rather than prevention of the syndrome, since the cause is unknown. The maternal mortality rate due to AFES has been reported to be anywhere from 10 to $90 \%,{ }_{11}^{11}$ although more recent data from large unselected population suggest that overall mortality rates may be closer to $20 \% .6,10$ Even those who survive generally have a poor outcome, with as many as $85 \%$ suffering significant neurologic injury due to cerebral hypoxia. ${ }^{6}$ Because the maternal and fetal mortality rates are so high, it is important to support the patient's family members. When the mother and infant are gravely ill, keeping their family members well informed and allowing as much access to the loved ones as possible are important. ${ }^{12}$

The initial management of amniotic fluid embolism relies on early suspicion and early aggressive hemodynamic support. Oxygenation, circulatory support, and correction of coagulopathy continue to be the mainstays of therapy. Specific laboratory coagulation abnormalities are treated with transfusion of fresh frozen plasma, platelets, cryoprecipitate. Hysterectomy may be required if there is persistent uterine hemorrhage to save the mother's life and should be decided early. ${ }^{8}$

Early and aggressive management of patients with clinically suspected AFE improves survival. It is important to always consider AFE in the differential diagnosis of sudden maternal cardiopulmonary instability. The input of consultants (anesthesiologist, obstetricians, hematologists, intensivists) must be enlisted early. ${ }^{9}$ AFE remains a diagnosis of exclusion and should always be considered early in the course of clinical management of any obstetric emergency involving cardiovascular collapse. Goal of therapy is to prevent additional hypoxia and end-organ hypoperfusion and organ system failure. 


\section{KNOWLEDGE GAP}

Our understanding of the pathogenesis and diagnosis of AFE is rudimentary. ${ }^{5}$ The pathophysiologic mechanisms responsible for this catastrophic process require further elucidation. ${ }^{6}$ The clinical diagnosis remains one of exclusion in combination with management of the presenting clinical symptoms.

\section{REFERENCES}

1. Meyer JR. Embolia pulmonar amnio caseosa. Bra Med 1926; (2):301-303.

2. Steiner PE, Lushbaugh CC. Maternal pulmonary embolism by amniotic fluid as a cause of obstetric shock and unexpected deaths in obstetrics. JAMA 1941;117:1245-1254, 1341-1345.

3. Kramer MS, et al. Maternal health study group of the Canadian perinatal surveillance system: amniotic-fluid embolism and medical induction of labour: a retrospective, populationbased cohort study. Lancet 2006;368(9545):1444-1448.

4. Abenhaim HA, Azoulay L, Kramer MS, Leduc L. Incidence and risk factors of amniotic fluid embolisms: a populationbased study on 3 million births in the United States. Am J Obstet Gynecol 2008 Jul;199(1):49.e1-8.
5. Knight M, Tuffnell D, Brocklehurst P, Spark P, Kurinczuk JJ. UK obstetric surveillance system: incidence and risk factors for amniotic-fluid embolism. Obstet Gynecol 2010 May;115(5): 910-917.

6. Lang CT, King JC. Maternal mortality in the United States. Best Pract Res Clin Obstet Gynaecol 2008 Jun;22(3):517-531.

7. Stroup DF, Berlin JA, Morton SC, et al. Meta-analysis of observational studies in epidemiology (MOOSE) group. JAMA 2000 Apr 19;283(15):2008-2012.

8. Clark SL, Belfort MA, Dildy GA, Herbst MA, Meyers JA, Hankins GD. Maternal death in the 21st century: causes, prevention, and relationship to cesarean delivery. Am J Obstet Gynecol 2008 Jul;199(1):36.e1-5.

9. Pallasmaa N, Ekblad U, Gissler M. Severe maternal morbidity and the mode of delivery. Acta Obstet Gynecol Scand 2008; 87(6):662-628.

10. Knight M, UKOSS. Amniotic fluid embolism: active surveillance versus retrospective database review. Am J Obstet Gynecol 2008 Oct;199(4):e9.

11. Zhu L, Qin M, Du L, et al. Comparison of maternal mortality between migrating population and permanent residents in Shanghai, China, 1996-2005. BJOG 2009 Feb;116(3):401-407.

12. Rudra A, Chatterjee S. Amniotic fluid embolism. Ind J Crit Care Med 2009 Jul-Sep;13(3):129-135. 